\title{
Time and Frequency Domain Simulation, Measurement and Optimization of Log-Periodic Antennas
}

\author{
Keyur K. Mistry ${ }^{1}$ (D) . Pavlos I. Lazaridis ${ }^{1} \cdot$ Zaharias D. Zaharis $^{2}$. \\ Mobayode O. Akinsolu ${ }^{3} \cdot$ Bo Liu $^{3} \cdot$ Thomas D. Xenos $^{2} \cdot$ Ian A. Glover ${ }^{1} \cdot$ Ramjee Prasad $^{4}$
}

Published online: 5 April 2019

(C) The Author(s) 2019

\begin{abstract}
Log-periodic antenna is a special antenna type utilized with great success in many broadband applications due to its ability to achieve nearly constant gain over a wide frequency range. Such antennas are extensively used in electromagnetic compatibility measurements, spectrum monitoring and TV reception. In this study, a log-periodic dipole array is measured, simulated, and then optimized in the $470-860 \mathrm{MHz}$ frequency band. Two simulations of the antenna are initially performed in time and frequency domain respectively. The comparison between these simulations is presented to ensure accurate modelling of the antenna. The practically measured realized gain is in good agreement with the simulated realized gain. The antenna is then optimized to concurrently improve voltage standing wave ratio, realized gain and front-to-back ratio. The optimization process has been implemented by using various algorithms included in CST Microwave Studio, such as Trusted Region Framework, Nelder Mead Simplex algorithm, Classic Powell and Covariance Matrix Adaptation Evolutionary Strategy. The Trusted Region Framework algorithm seems to have the best performance in adequately optimizing all predefined goals specified for the antenna.
\end{abstract}

Keywords Boom · CST · Dipoles · Directivity · Electromagnetic compatibility · Evolutionary algorithms $\cdot$ Gain $\cdot \log$-periodic antenna $\cdot$ LPDA $\cdot$ Measurements · Optimization $\cdot$ TV reception $\cdot$ UHF

\section{Introduction}

Log-periodic antennas are widely used because of their broadband characteristics in TV reception, electromagnetic compatibility measurements and wideband precision measurements. Log-periodic dipole arrays (LPDAs) present an almost flat gain over a wide

Keyur K. Mistry

keyur.mistry@hud.ac.uk

1 University of Huddersfield, Queensgate, Huddersfield HD1 3DH, UK

2 Aristotle University of Thessaloniki, 54124 Thessaloniki, Greece

3 Wrexham Glyndwr University, Wrexham LL11 2AW, UK

4 Department of Business Development and Technology, Aarhus University, 7400 Herning, Denmark 
operating bandwidth [1]. The gain of the antenna can be increased by increasing the number of dipoles [2]. LPDAs provide better front-to-back ratio but relatively lower gain than YagiUda array antenna [3]. However, the LPDA has much larger bandwidth compared to YagiUda antenna. Furthermore, the most important difference between the two antennas lies in their feeding patterns. Each dipole of LPDA is connected to the feeding source, whereas in Yagi-Uda antenna only one dipole is connected to the feeding source and all other dipoles are passive [4]. A very useful design procedure of LPDA has been proposed by Carrel [5, 6].

The LPDA is designed by using several dipoles of different lengths. In this way, each dipole operates in resonance condition at a certain frequency and this happens when the dipole length $\mathrm{L}$ is equal to half wavelength $(\lambda / 2)$. At the same time, dipoles with length greater or less than $\lambda / 2$ at the same frequency act respectively as reflectors or directors, since they are away from their resonance condition. By employing dipoles of varying lengths, the LPDA is capable of operating effectively at a wide frequency range [4].

Figure 1 presents the basic geometry of a log-periodic dipole antenna. The LPDA consists of several parallel wire dipoles of varying lengths, which are arranged in a sequence so as to be included within the same angle $2 \alpha$. The angle of intersection can be defined as:

$$
\alpha=\tan ^{-1}\left[\frac{1-\tau}{4 \sigma}\right]
$$

where $\tau$ is a scaling factor defined as the ratio of the lengths or diameters of two consecutive dipoles, as given by

$$
\tau=\frac{L_{n+1}}{L_{n}}=\frac{d_{n+1}}{d_{n}}
$$

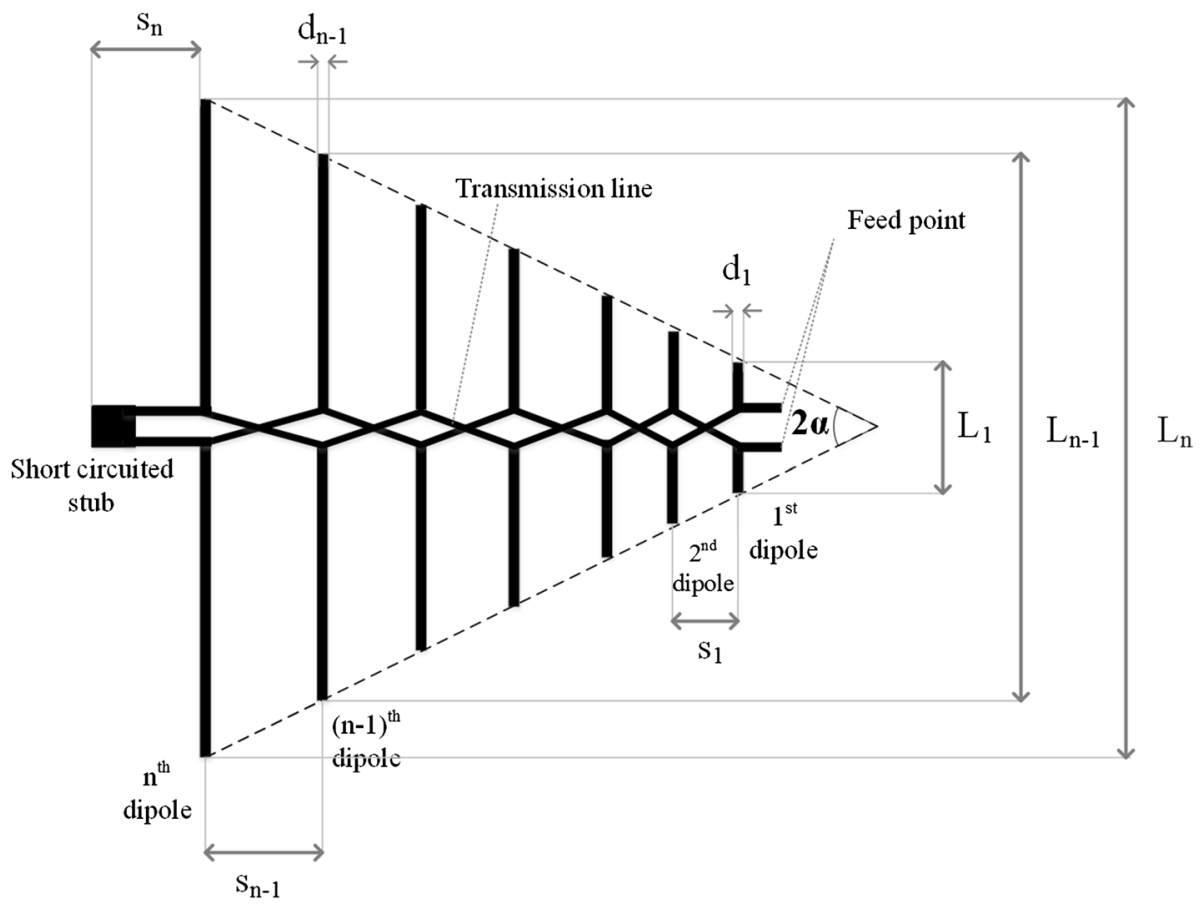

Fig. 1 Log-periodic dipole array geometry 
and $\sigma$ is a spacing factor constant defined by

$$
\sigma=\frac{s_{n}}{2 L_{n}}
$$

where $L_{n}$ and $d_{n}$ are respectively the length and the diameter of the nth dipole, while $s_{n}$ is the spacing between the nth dipole and its consecutive $(n+1)$ th dipole.The dipoles are connected to the feeding line in such a way that a phase reversal is obtained in the feeding between two consecutive dipoles [7]. In some LPDA designs, constant diameter dipoles are used to reduce cost [8]. The values of $\tau$ and $\sigma$ are properly chosen by the user to design the antenna with predefined average directivity over the operating bandwidth, and this is done by using the well-known Carrel's graph $[5,9,10]$.

\section{Time Domain and Frequency Domain Simulation}

The selection of the solver for the simulation of an antenna is a challenge for many researchers. There is no best suited solver for all types of applications. The time domain solver in the CST Microwave Studio (MWS) is based on the finite integration technique (FIT) describing Maxwell's equations on a time-grid space whereas the frequency domain solver is based on the finite element method (FEM). The time domain method is more suitable for calculations over a wide frequency range because only one simulation is required and afterwards the frequency response is obtained through Fast Fourier Transform (FFT). Another interesting application of the time domain solver is that the calculation of the spectrum can be performed with an arbitrarily fine frequency resolution without any additional effort. Therefore, the time domain solver is more suitable for simulations of electrically large structures. The smaller the mesh cells are (more mesh cells), the longer the calculation time will be for this solver. The time domain solver in CST uses a hexahedral or a hexahedral transmission-line matrix (TLM) meshing technique for the simulation. The time domain simulation analysis is performed on a port-by-port basis.

On the other hand, when the frequency domain method is used, discrete simulations must be performed at discrete frequencies separated from each other by a specified frequency step to cover the entire operating bandwidth. Thus, adaptive refinement of the mesh at every frequency can be performed to obtain the results. The frequency domain simulation stops when the S-parameters converge. Nevertheless, a single simulation can provide results for all the ports in a single calculation. Therefore, the frequency domain solver finds its application in simulations of narrowband and electrically small structures. In CST, this solver uses either the hexahedral or tetrahedral meshing technique [11].

\section{LPDA Measurments and Simulations}

This study presents the measurement results of a ten-element LPDA conducted in open field conditions in 470-860 MHz frequency band (former analogue UHF TV band). The measurements are performed using a Rohde and Schwartz FSH8 portable spectrum analyzer. The gain of the antenna is measured using the reference antenna method with the help of calibrated biconical dipole antennas. A model of this antenna is developed in CST MWS 2016 to compare the simulation results with the measured results of the antenna. The simulation results obtained from CST MWS include the VSWR, realized gain and 
front-to-back ratio versus frequency. The realized gain of the antenna is defined as the difference between gain and mismatch loss of the antenna. The expressions for realized gain and mismatch loss are given below:

$$
\begin{aligned}
& \text { Mismatch Loss }(d B)=-10 \log _{10}\left[1-\left[\frac{V S W R-1}{V S W R+1}\right]^{2}\right] \\
& \text { Realized Gain }(\mathrm{dBi})=\text { Gain }(\mathrm{dBi})-\text { Mismatch loss }(\mathrm{dB})
\end{aligned}
$$

Furthermore, the optimization of this antenna has been performed in CST MWS by using Trusted Region Framework, Nelder Mead simplex algorithm, Classic Powell and Covariance Matrix adaptation evolutionary strategy (CMA-ES) included in this simulator.

Figure 2 presents an actual LPDA (left) and a designed model of the same LPDA in CST MWS (right). Both the antenna and its CST model include a short-circuited stub at their rear end. The CST model is discretized using hexahedral meshing with 564,435 cells. All simulations are performed with $-50 \mathrm{~dB}$ accuracy and with a port impedance of $50 \Omega$. The simulation of the antenna model is performed in time domain and frequency domain. Figures 3, 4 and 6 show respectively the comparative results of VSWR, realized gain and front-to-back ratio.

After this preliminary check of the antenna model, there is confidence that the results from time and frequency domain simulations are correct due to their good agreement. Also, the difference between the simulated and the measured realized gain is less than $0.5 \mathrm{~dB}$ in the frequency range of $490-860 \mathrm{MHz}$ as shown in Fig. 5. Therefore, the CST model approximates with good accuracy the actual antenna. Optimization of this antenna is then performed using various optimization algorithms included in CST MWS.

\section{LPDA Optimization}

CST includes global optimizers and local optimizers. However, the search capability is larger in global optimizers compared to local optimizers. Trusted Region Framework (TRF), Nelder Mead simplex algorithm and Classic Powell algorithm are examples of local optimizers. On the other hand, CMA-ES is an example of a global optimizer.
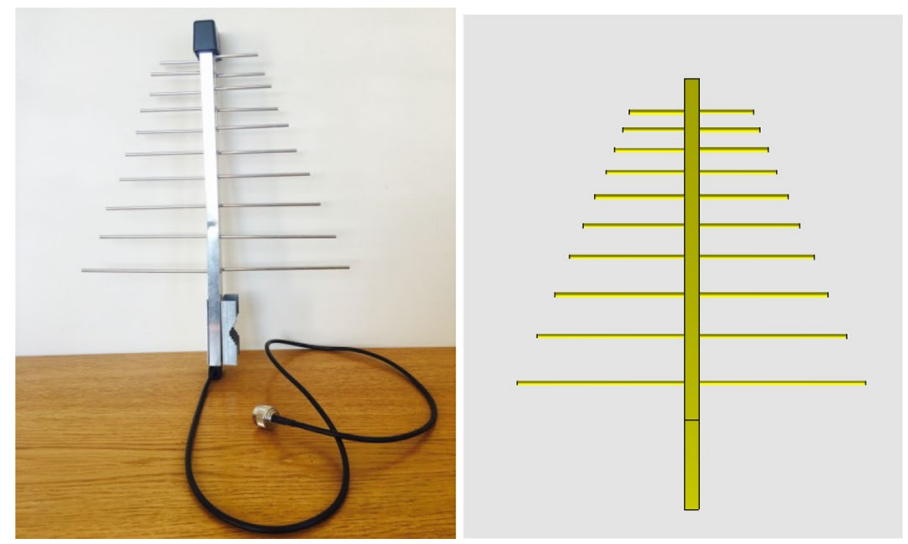

Fig. 2 Actual ten-element LPDA (left) and LPDA model in CST MWS (right) 


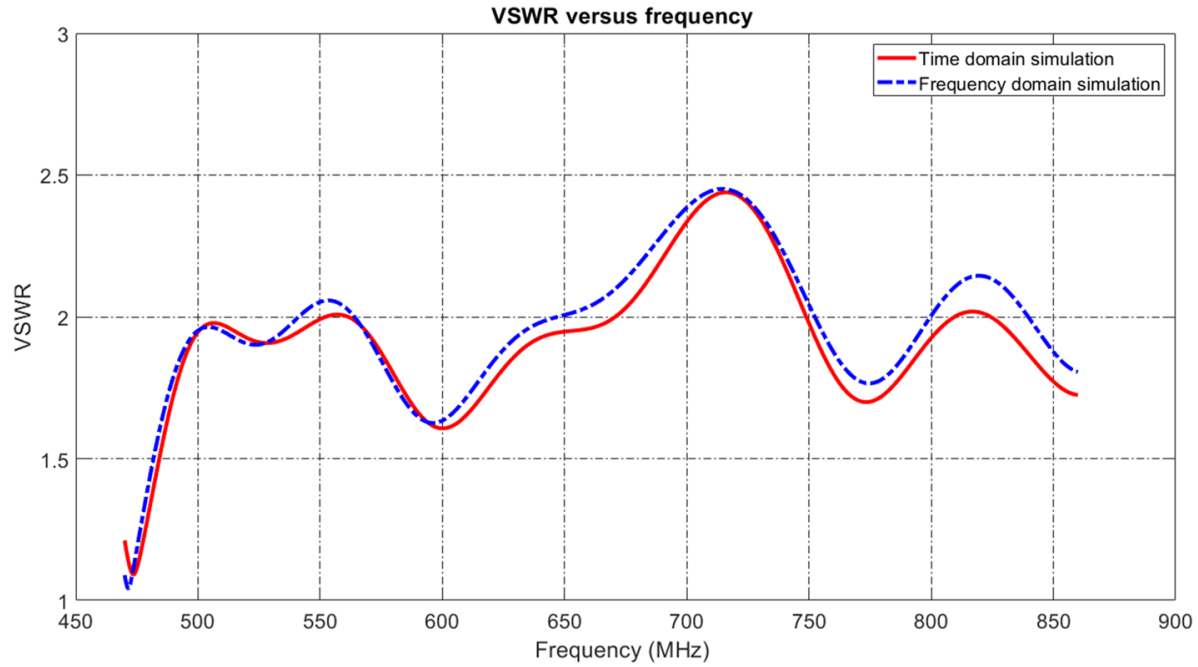

Fig. 3 Comparison between VSWR values derived from simulations in time and frequency domain. (Color figure online)

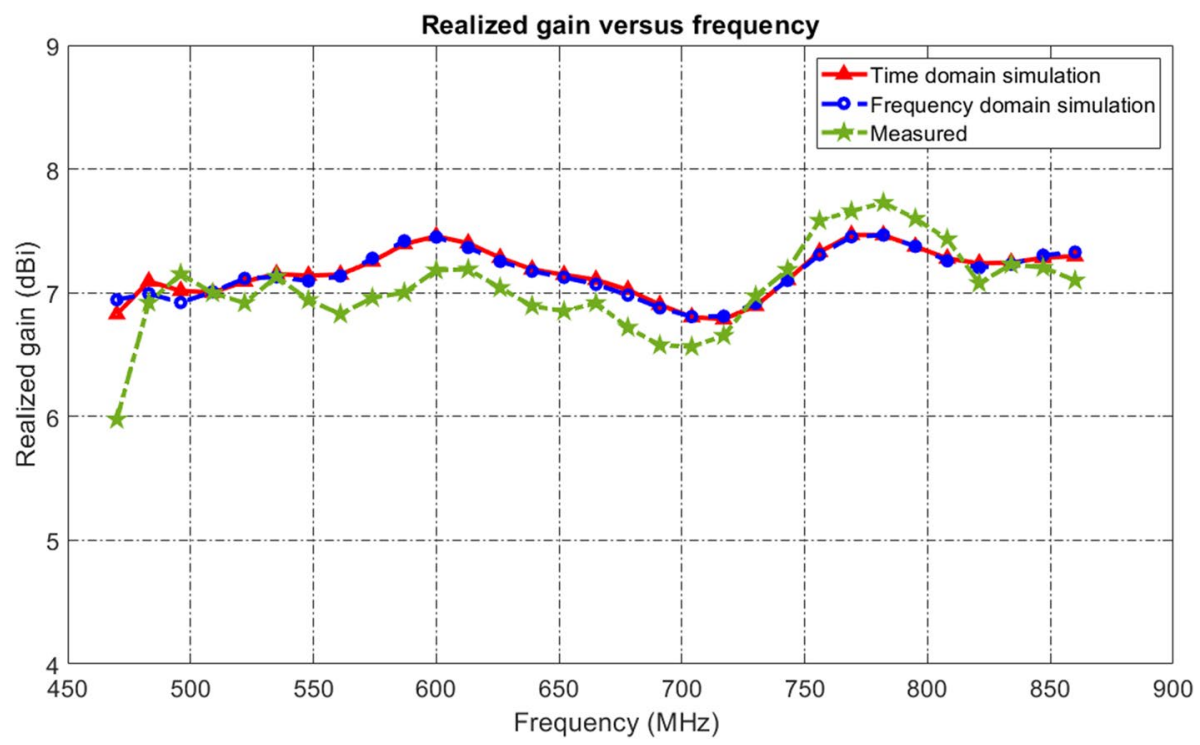

Fig. 4 Comparison between realized gain values derived from simulations in the time and in the frequency domain and open-field measurements (green line). (Color figure online)

In TRF optimizer, a linear model is created based on the primary data collected from a trust region around the starting point. The new model then acts as a new starting point until it converges to obtain an accurate model of the data. It is one of the most robust optimization algorithms offered by CST. TRF algorithm has a unique ability to predict improved fitness values by adjusting the limits and providing global convergence [12]. Nelder and Mead (in 1965) introduced Nelder Mead Simplex search algorithm, which is an improved version of the Simplex search algorithm proposed by Spendley, Hext and Himsworth in 


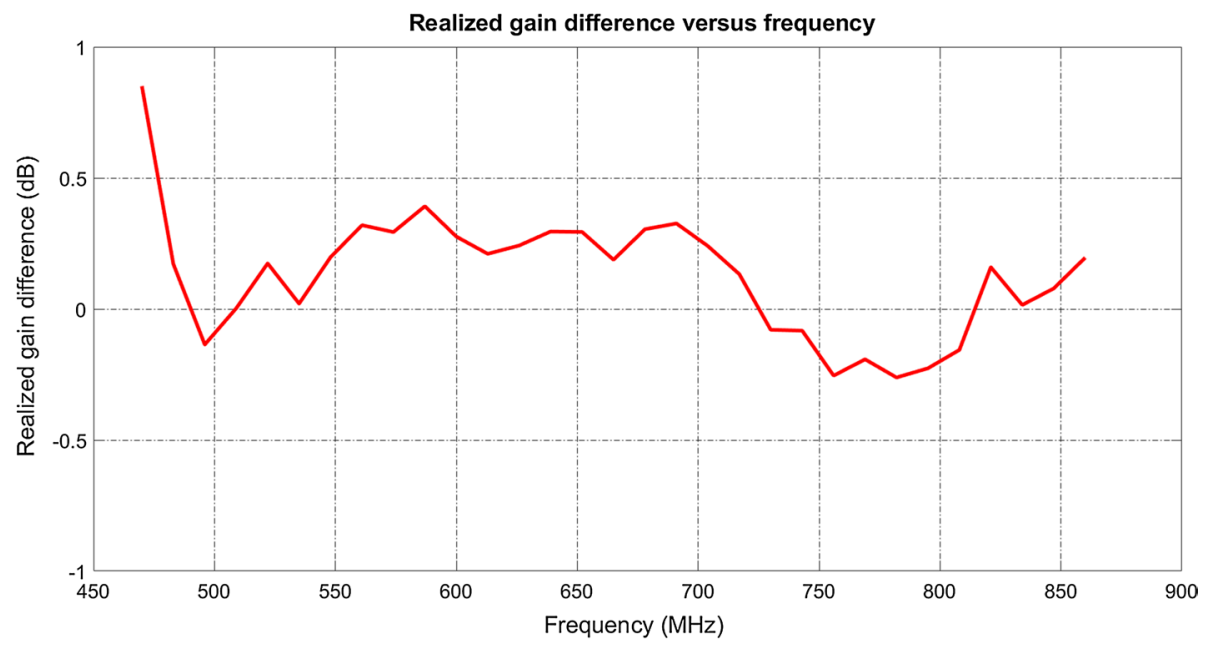

Fig. 5 Difference of simulated realized gain (time domain) and measured realized gain versus frequency

1962 [13]. This algorithm utilizes multiple points, which are distributed across the parameter search space, so that the optimum value can be identified. It can be used as a solution for complex problem domains consisting of relatively few parameters. Classic Powell algorithm is considered to be a simple and robust optimizer, which can be used for a problem involving few parameters. The time of termination of the optimization process is decided by the accuracy requirement set before the start of the process. It is a relatively slow algorithm compared to other algorithms. However, it provides accurate results in some cases. CMA-ES is a self-adaptive evolution strategy and a global optimizer, which was developed by Hansen and Ostermeier, [14]. CMA-ES initializes strategy parameters like the number of variables, population size and their bounds in a well-defined fashion and thus does not require parameter tuning by the user $[14,15]$. This algorithm is based on the evolution of a population of individuals. A comparison of CMA-ES with particle swarm optimization (PSO) is presented in [16]. CMA-ES solves a problem by generating a population of individuals using a Gaussian distribution [15, 17].

TRF algorithm, Nelder Mead simplex algorithm, Classic Powell and CMA-ES are used here to optimize the LPDA so that the antenna satisfies the requirements stated in Table 1. The half-dipole lengths ( $\mathrm{Ln} / 2)$, radius of the dipoles ( $\mathrm{rad}$ ), gap between the boom (gap), length of the boom (1_boom), width of the boom (b_boom), height of the boom (h_boom), length of the connector (1_connector) and spacing between the dipoles (sn) are the geometry parameters to be considered while performing optimization.

The aim of the above-mentioned algorithms is to find the minimum value of a fitness function, so that all goals are satisfied. Therefore, the fitness function is constructed as a linear combination of the parameters to be optimized by taking into account the respective weights shown in Table 1. Then the global minimum of the fitness function is achieved by the algorithms by selecting proper values for the geometry parameters mentioned above. The optimized results of VSWR, realized gain and front-to-back ratio obtained with various evolutionary algorithms are shown respectively in Figs. 6, 7 and 8. In these figures, the results of the initial CST antenna model are also displayed.

From Fig. 7, it is evident that the best performance in minimizing VSWR is obtained from TRF algorithm followed by the Nelder Mead Simplex algorithm. TRF algorithm is 
Table 1 Goals and weights for the antenna optimization

\begin{tabular}{|c|c|c|c|}
\hline Parameter & Operator & Goal value & Weight \\
\hline VSWR & $<$ & 1.5 & 10 \\
\hline Gain (dBi) & $>$ & 8 & 0.5 \\
\hline $\begin{array}{l}\text { Front-to-back ratio } \\
(\mathrm{dB})\end{array}$ & $>$ & 20 & 0.2 \\
\hline
\end{tabular}

\section{Front-to-back ratio versus frequency}

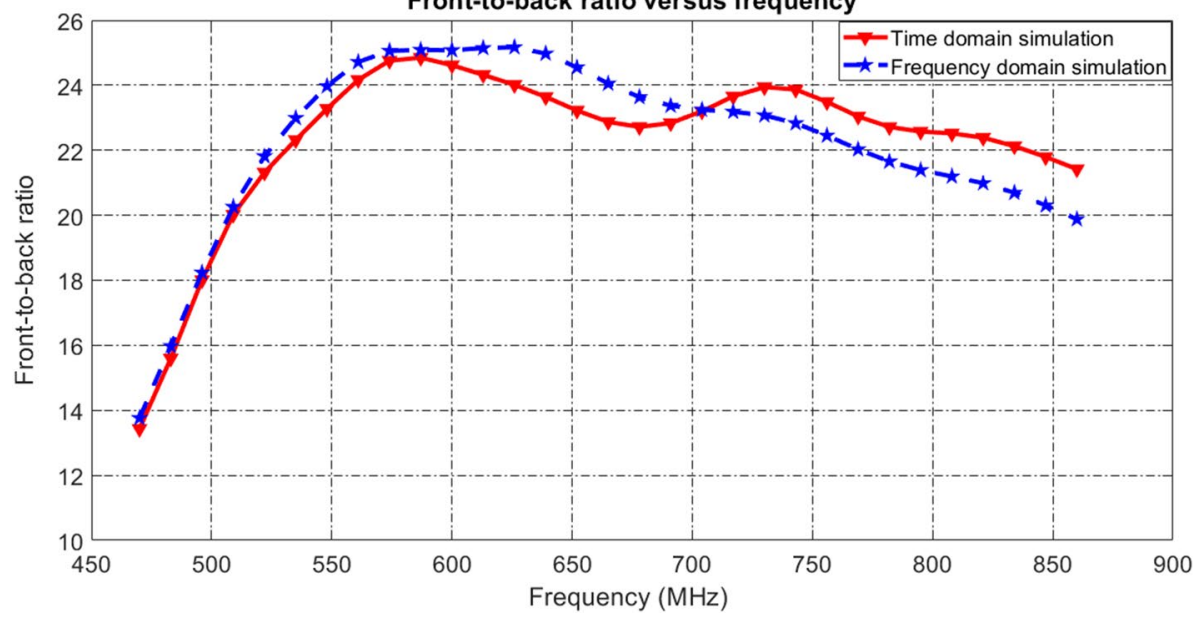

Fig. 6 Comparison between front-to-back ratio values $(\mathrm{dB})$ derived from simulations in time and frequency domain. (Color figure online)

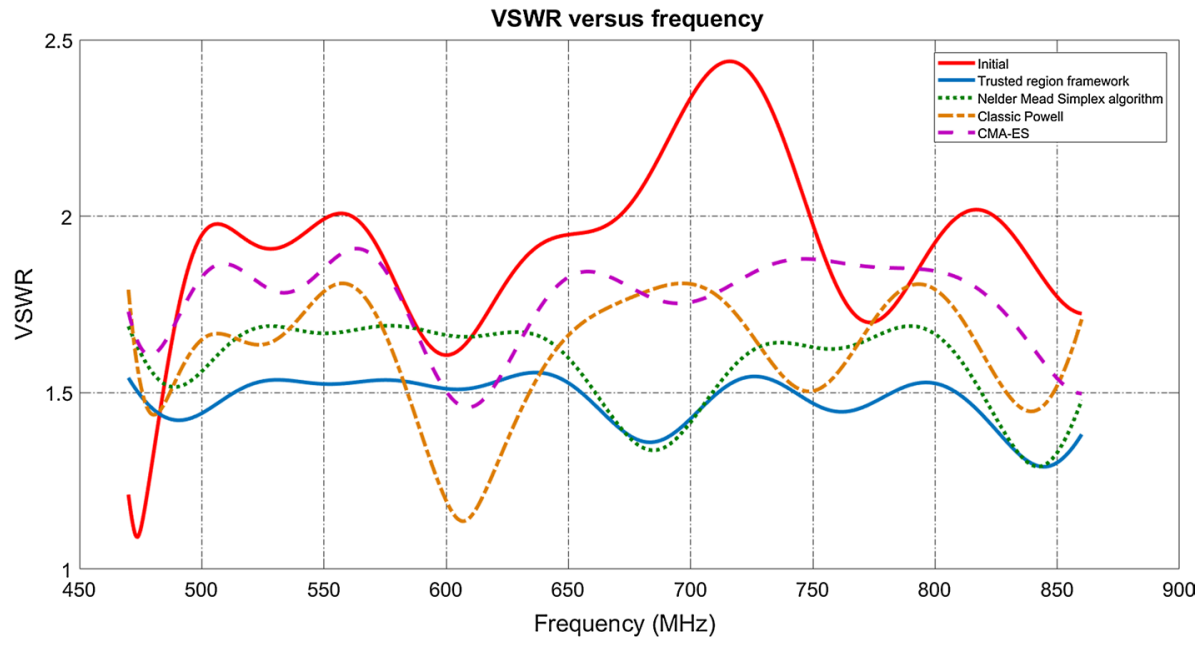

Fig. 7 Comparison between optimized and initial VSWR values 


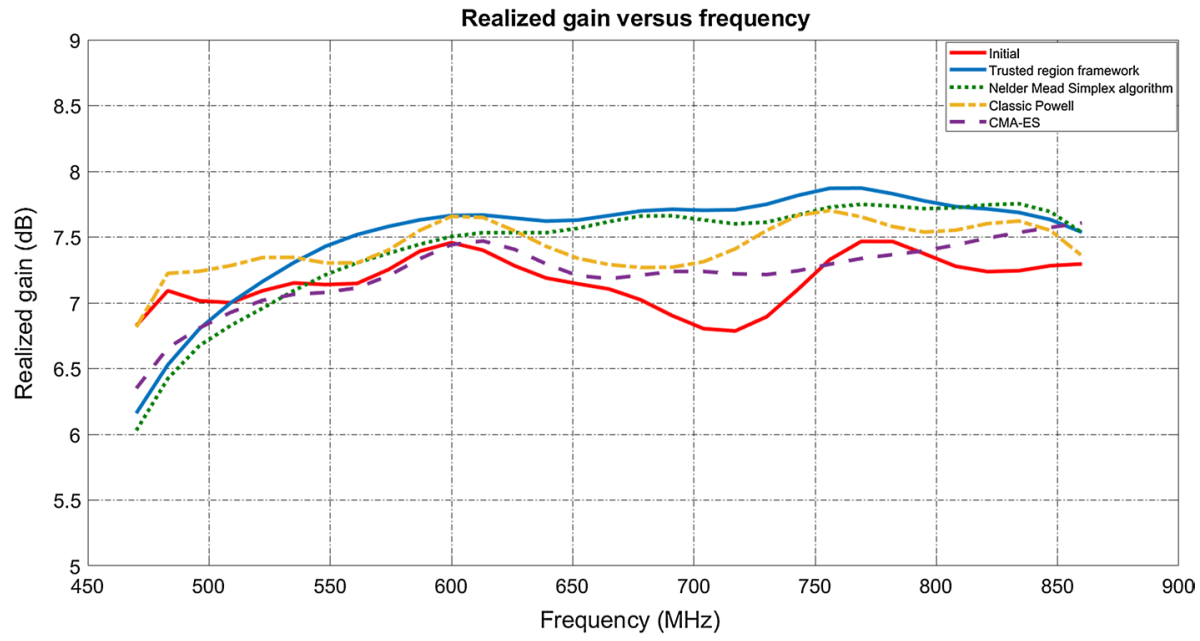

Fig. 8 Comparison between optimized and initial realized gain

successful in minimizing VSWR to approximately 1.5. Other algorithms obtain VSWR values oscillating between 1.5 and 2. CMA-ES algorithm exhibits the poorest performance with the highest VSWR.

As shown in Fig. 8, the best optimizer of realized gain is again TRF algorithm since it produces a relatively flat realized gain approximately equal to $7.8 \mathrm{dBi}$. The other algorithms exhibit an average performance by obtaining a flat realized gain between 7 and 7.8 $\mathrm{dBi}$ above $550 \mathrm{MHz}$. Thus, an important improvement of approximately $1 \mathrm{~dB}$ is observed between the initial and the optimized realized gain by TRF algorithm in the frequency range of $670-750 \mathrm{MHz}$. On the other hand, the lowest realized gain is again obtained by CMA-ES algorithm.

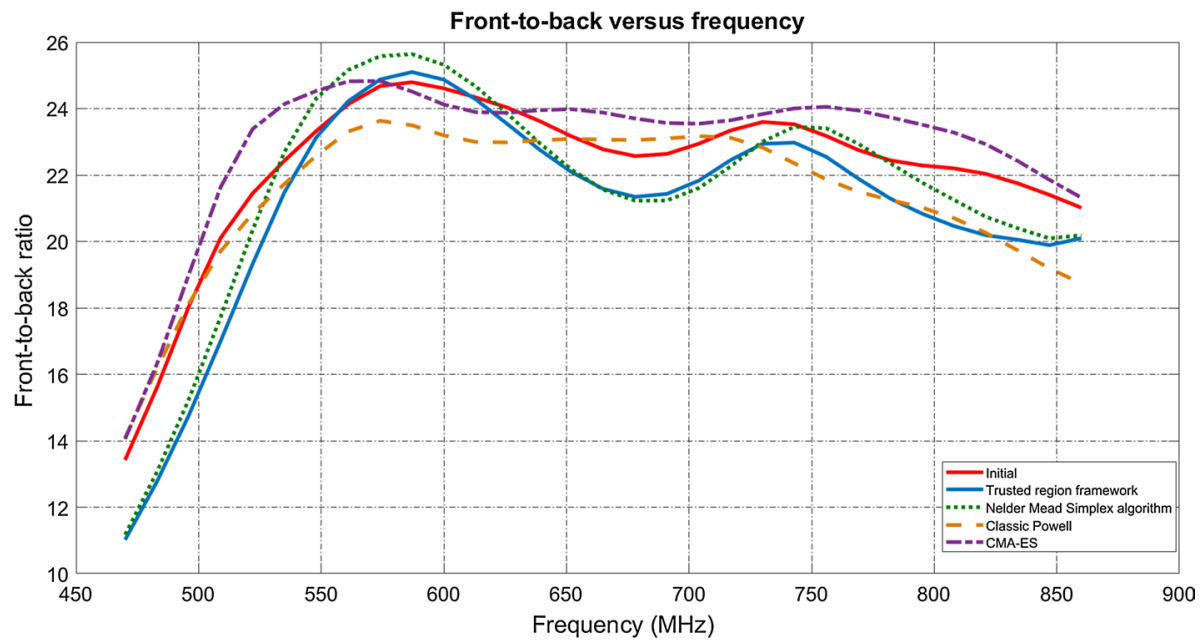

Fig. 9 Comparison between optimized and initial front-to-back ratio 
Table 2 LPDA geometry before and after optimization (dimensions in $\mathrm{cm}$ )

\begin{tabular}{|c|c|c|c|c|c|}
\hline Parameters & Initial & TRF & Nelder mead & Classic Powell & CMA-ES \\
\hline b_boom & 1.30 & 1.42 & 1.41 & 1.30 & 1.30 \\
\hline h_boom & 1.30 & 1.43 & 1.40 & 1.43 & 1.24 \\
\hline 1_boom & 30.60 & 32.58 & 32.25 & 29.30 & 30.10 \\
\hline gap & 1.10 & 0.99 & 1.02 & 1.03 & 1.06 \\
\hline 1_connector & 8.00 & 8.56 & 8.26 & 8.30 & 8.11 \\
\hline L1 & 9.80 & 10.76 & 10.78 & 10.74 & 9.90 \\
\hline $\mathrm{L} 2$ & 11.00 & 12.1 & 12.06 & 12.04 & 11.46 \\
\hline L3 & 12.40 & 11.84 & 12.22 & 12.40 & 12.12 \\
\hline L4 & 14.00 & 13.72 & 13.94 & 13.96 & 13.22 \\
\hline L5 & 16.00 & 15.82 & 15.80 & 16.00 & 15.46 \\
\hline L6 & 18.00 & 17.76 & 17.84 & 17.96 & 17.78 \\
\hline L7 & 20.60 & 19.82 & 19.88 & 20.58 & 20.5 \\
\hline L8 & 23.20 & 22.46 & 22.68 & 23.16 & 23.20 \\
\hline L9 & 26.40 & 25.76 & 26.16 & 26.34 & 26.70 \\
\hline L10 & 29.80 & 31.30 & 32.54 & 29.92 & 31.24 \\
\hline $\mathrm{rad}$ & 0.20 & 0.22 & 0.21 & 0.22 & 0.19 \\
\hline s0 & 3.00 & 2.72 & 2.71 & 2.70 & 2.84 \\
\hline s1 & 1.60 & 1.45 & 1.55 & 1.44 & 1.53 \\
\hline s2 & 1.80 & 1.77 & 1.91 & 1.68 & 1.82 \\
\hline s3 & 2.00 & 2.05 & 2.05 & 1.91 & 2.05 \\
\hline s4 & 2.20 & 2.41 & 2.37 & 2.41 & 2.23 \\
\hline s5 & 2.60 & 2.49 & 2.34 & 2.58 & 2.69 \\
\hline s6 & 2.80 & 2.82 & 2.72 & 2.52 & 2.82 \\
\hline s7 & 3.40 & 3.10 & 3.06 & 3.07 & 3.51 \\
\hline s8 & 3.70 & 3.68 & 3.81 & 3.99 & 3.69 \\
\hline s9 & 4.20 & 4.00 & 3.78 & 4.48 & 4.20 \\
\hline
\end{tabular}

In Fig. 9, CMA-ES algorithm seems to be the best performer in maximizing the frontto-back ratio above $20 \mathrm{~dB}$ followed by Nelder Mead Simplex algorithm and TRF algorithm. However, all algorithms have satisfactorily met the goal $(>20 \mathrm{~dB})$ and show approximately similar front-to-back ratio, oscillating between 21 and $24 \mathrm{~dB}$. The optimal LPDA dimensions derived from the above-mentioned optimizers and the initial antenna dimensions are shown in Table 2. The optimization algorithms have been configured to change the antenna dimensions only by $\pm 10 \%$ from their initial values.

\section{Conclusion}

The accurate modelling and simulation of a ten-dipole LPDA has been successfully performed using the time domain and frequency domain solvers of CST MWS. The simulated and practically measured results have been compared to ensure validity of the CST model. A comparative study of optimization of LPDA using various optimization algorithms included in CST MWS has been performed to obtain the best results for VSWR, 
realized gain and front-to-back ratio. TRF algorithm demonstrated the fastest convergence and achieved the best overall results with the best fitness function value.

Open Access This article is distributed under the terms of the Creative Commons Attribution 4.0 International License (http://creativecommons.org/licenses/by/4.0/), which permits unrestricted use, distribution, and reproduction in any medium, provided you give appropriate credit to the original author(s) and the source, provide a link to the Creative Commons license, and indicate if changes were made.

\section{References}

1. Zaharis, Z. D., Skeberis, C., Xenos T. D., Lazaridis, P. I., \& Stratakis, D. I. (2014). IWO-based synthesis of log-periodic dipole array. In International Conference on Telecommunications and Multimedia (TEMU), Heraklion (pp. 150-154).

2. Lazaridis, P., Tziris, E., Zaharis, Z., Xenos, T., Cosmas, J., Gallion, P., et al. (2016). Comparison of evolutionary algorithms for LPDA antenna optimization. Radio Science, 51(8), 1377-1384.

3. Casula, G., Maxia, P., Mazzarella, G., \& Montisci, G. (2013). Design of a printed log-periodic dipole array for ultra-wideband applications. Progress In Electromagnetics Research C, 38, 15-26.

4. Balanis, C. A. (1997). Antenna theory, analysis and design (2nd ed., pp. 551-566). New York: Wiley.

5. Carrel, R.L. (1961)Analysis and design of the Log-periodic dipole antenna. Technical Report No. 52, Electrical Engineering Department, University of Illinois.

6. Huang, Y., \& Boyle, K. (2008). Antennas (1st ed.). Chichester: Wiley.

7. Mistry, K., Lazaridis, P., Zaharis, Z., Xenos, T., \& Glover, I. (2017). Optimization of log-periodic dipole antenna with LTE bandrejection. In: Loughborough Antenna Propagation Conference (LAPC), Loughborough.

8. Zaharis, Z., Skeberis, C., Lazaridis, P., \& Xenos, T. (2016). Optimal wideband LPDA design for efficient multimedia content delivery over emerging mobile computing systems. IEEE Systems Journal, 10(2), 831-838.

9. Karim, M. A., Rahim, M., Majid, H., Ayop, O., Abu, M., \& Zubir, F. (2010). Log periodic fractal Koch antenna for the UHF band applications. Progress In Electromagnetics Research, 100, 201-218.

10. Lazaridis, P., Zaharis, Z., Skeberis, C., Xenos, T., Tziris, E., \& Gallion, P. (2014). Optimal design of UHF TV band log-periodic antenna using invasive weed optimization. In: 4th International Conference on Wireless Communications, Vehicular Technology, Information Theory and Aerospace and Electronic Systems (VITAE).

11. Weiland, T., Timm, M., \& Munteanu, I. (2008). A practical guide to 3-D simulation. IEEE Microwave Magazine, 9(6), 62-75.

12. Lim, D., Ong, Y.-S., Jin, Y., \& Sendhoff, B. (2006). Trusted evolutionary algorithm. In IEEE International Conference on Evolutionary Computation (pp. 149--156).

13. Xu, S., Zou, X., Liu, W., Wang, X., Zhu, H., \& Zhao, T. (2010). Research of particle swarm optimization algorithm based on Nelder-Mead simplex and its application on partial discharge parameter recognition. In IEEE International Power Modulator and High Voltage Conference, Atlanta, GA (pp. 719-722).

14. Hansen, N., \& Ostermeier, A. (2001). Completely derandomized self-adaptation in evolutionary strategies. Evolutionary Computation, 9(2), 159-195.

15. BouDaher, E., \& Hoorfar, A. (2015). Electromagnetic optimization using mixed-parameter and multiobjective covariance matrix adaptation evolution strategy. IEEE Transactions on Antennas and Propagation, 63(4), 1712-1724.

16. Kovitz, J.M., \& Rahmat-Samii, Y. (2013). A comparative study between CMA evolution strategies and particle swarm optimization for antenna applications. In US National Committee of URSI National Radio Science Meeting (USNC-URSI NRSM), Boulder, CO (pp. 1-1).

17. Awotunde, A. (2015). Estimation of well test parameters using global optimization techniques. Journal of Petroleum Science and Engineering, 125, 269-277.

Publisher's Note Springer Nature remains neutral with regard to jurisdictional claims in published maps and institutional affiliations. 

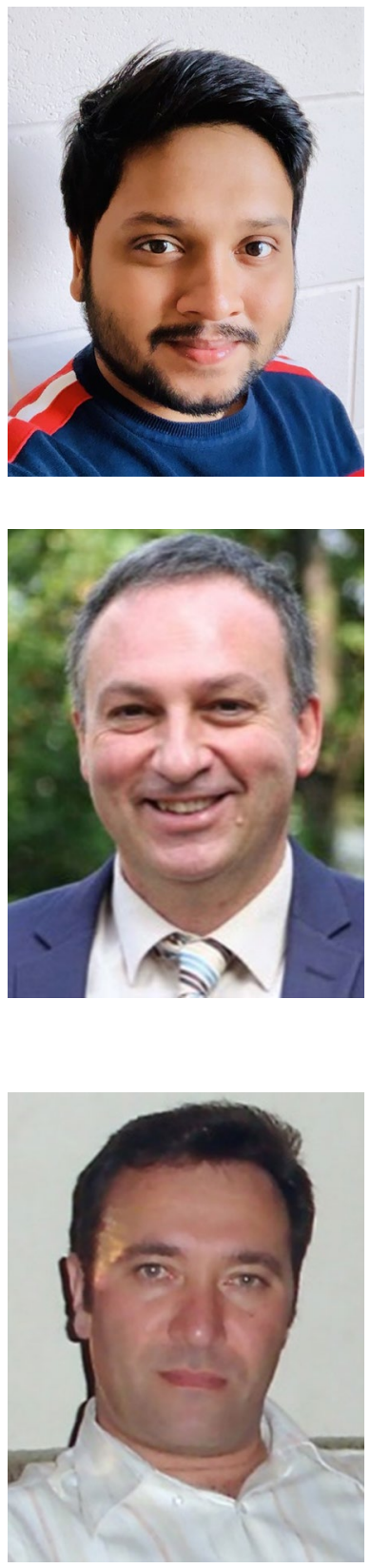

Keyur K. Mistry was born in Mumbai, India in 1993. He received the B.Tech degree in electronics and telecommunication engineering from the NMIMS University, Mumbai, India in 2015 and the Master's degree in electronics and communications engineering from the University of Huddersfield in 2016. He is currently working as Ph.D. researcher in electronics and communications engineering. His research interests include antenna design and measurements, wireless partial discharge sensors, UHD TV broadcasting and developing optimization algorithms. He was awarded with Vice-Chancellor scholarship by the University of Huddersfield for his Ph.D. degree. He was also recipient of the 1 st prize for Best design for proof of concept design competition organized by ICE/Honeypot in 2016.

Pavlos I. Lazaridis received the B.Sc. degree in Electrical Engineering from Aristotle University of Thessaloniki, Greece, in 1990, the M.Sc. in Electronics from Université Pierre \& Marie Curie, Paris 6, France in 1992 and the Ph.D. from ENST Paris and Paris, in 1996. From 1991 to 1996, he was involved with research on semiconductor lasers and wave propagation for France Télécom and teaching at ENST Paris. In 1997, he became Head of the Antennas and Propagation Laboratory, TDFC2R Metz (Télédiffusion de France/France Télécom Research Center). From 1998 to 2002 he was senior Examiner at the European Patent Office (EPO), Den Haag, the Netherlands. From 2002 to 2014 he was involved with teaching and research at the ATEI of Thessaloniki, Greece and Brunel University West London. He is currently a Reader in Electronic and Electrical Engineering at the University of Huddersfield, United Kingdom, member of the IET, Senior Member IEEE, and a Fellow of the Higher Education Academy.

Zaharias D. Zaharis received the B.Sc. degree in Physics in 1987, the M.Sc. degree in Electronics in 1994, and the Ph.D. degree in 2000 from Aristotle University of Thessaloniki. Also, in 2011 he obtained the Diploma degree in Electrical and Computer Engineering from the same university. From 2002 to 2013, he has been working in the administration of the telecommunications network of Aristotle University of Thessaloniki. Since 2013, he is with the Department of Electrical and Computer Engineering, Aristotle University of Thessaloniki. His research interests include design and optimization of antennas and microwave circuits, mobile communications, radio-wave propagation, RF measurements, evolutionary optimization, neural networks, and signal processing. Dr. Zaharis is a member of the Technical Chamber of Greece and a senior member of IEEE. 

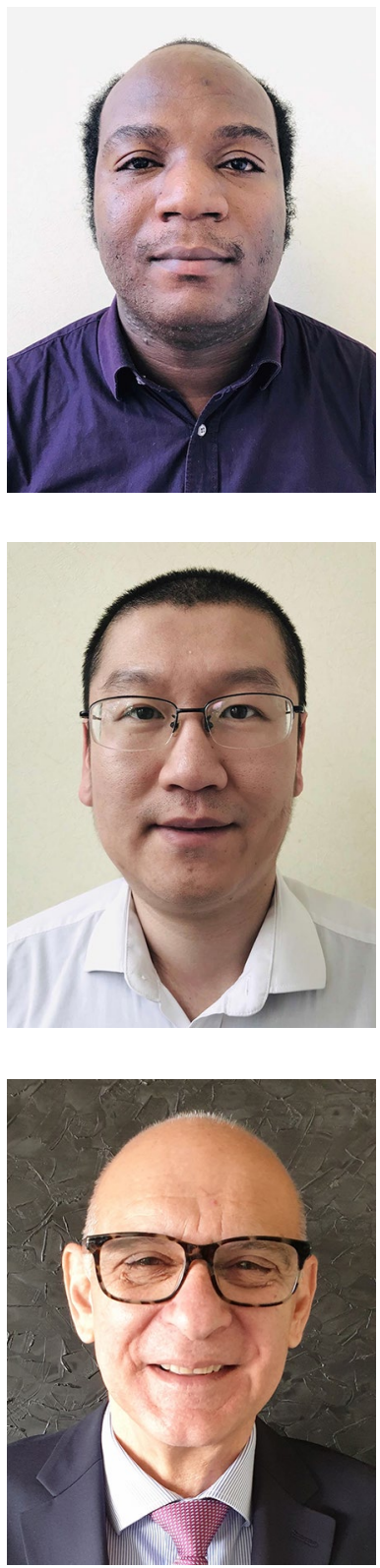

Mobayode 0. Akinsolu received the M.Sc. with distinction in Electrical and Electronic Engineering from University of Bradford in 2014. He then worked as a research fellow (industrial attaché) and a visiting researcher at the National Space Research and Development Agency, Nigeria and the RFID Research Centre, African University of Science and Technology, Nigeria respectively until 2016. In 2016, he became a Ph.D. scholarship awardee in recognition of a joint project between Wrexham Glyndwr University, UK and University of Birmingham, UK. His Ph.D. research focuses on electromagnetic design automation using surrogate model assisted evolutionary algorithms. He is a member of the IET and a member of the IEEE.

Bo Liu received the B.S. degree from Tsinghua University, P. R. China, in 2008. He received his Ph.D. degree at the MICAS laboratories of the University of Leuven (KU Leuven), Belgium, in 2012. From 2012 to 2013 , he was a Humboldt research fellow and was working with Technical University of Dortmund, Germany. In 2013, he was appointed lecturer at Wrexham Glyndwr University, UK, where he was promoted to Reader in 2016. He is an honorary fellow at The University of Birmingham. His research interests lie in artificial intelligencedriven design automation methodologies of analog/RF integrated circuits, microwave devices, MEMS, evolutionary computation and machine learning. He has authored or coauthored 1 book and more than 40 papers in international journals, edited books and conference proceedings. He is a senior member of IEEE.

Thomas D. Xenos was born in Thessaloniki, Greece, in 1955. He received the Diploma in Electrical Engineering from the University of Patras, Patras, Greece, in 1978 and the Ph.D. degree in wireless communications from the Aristotle University of Thessaloniki, Thessaloniki, in 1991. Since then, he has joined the Department of Electrical and Computer Engineering, Aristotle University of Thessaloniki, where he is currently a Professor. He has participated in many national and international projects. He is the author of over 85 scientific papers. His research interests include wireless communications, radiowave propagation, nonionizing electromagnetic radiation measurements, and electromagnetic compatibility. 


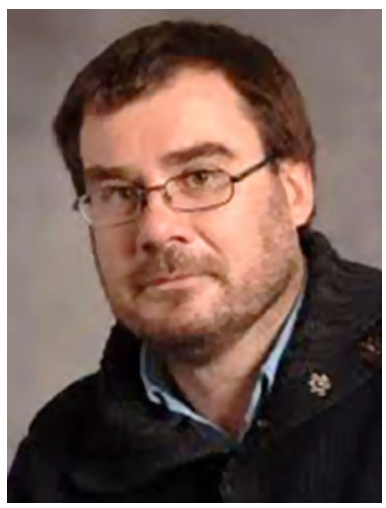

Ian A. Glover is a Radio Scientist and Wireless Communications Engineer. He is currently Professor of Radio Science and Wireless System Engineering, and Head of the Department of Engineering and Technology, at the University of Huddersfield in the UK. He is also Visiting Professor of Radio Science at the Universidade Federal de Campina Grande in Brazil. He has previously held senior academic posts at the Universities of Strathclyde, Bath and Bradford. Ian's principal current research interest is in the application of radiometric and wireless communication methods to insulation condition monitoring and asset management of high-voltage plant in the future smart grid. His other interests are in classical radio propagation for applications ranging from satellite communication, terrestrial microwave radio relay, mobile communications, radar and wireless sensor networks. He is the Chair of the UK Panel of the International Union of Radio Science (URSI) and is a past Associate Editor of the Radio Science Bulletin. He is the author, with Peter Grant, of Digital Communications (1998, 2004, 2008) published by Pearson and the editor (with Peter Shepherd and Stephen Pennock) of Microwave Devices, Circuits and Subsystems for Communications Engineering (2005) published by Wiley. Ian Glover is a member of the IET, IEEE and IoP, and is a Fellow of the Academy of Higher Education.

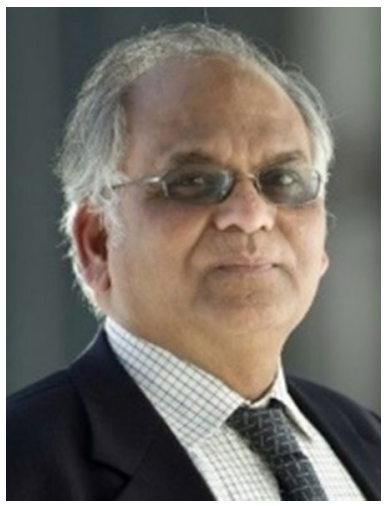

Ramjee Prasad is a Professor of Future Technologies for Business Ecosystem Innovation (FT4BI) in the Department of Business Development and Technology, Aarhus University, Denmark. He is the Founder President of the CTIF Global Capsule (CGC). He is also the Founder Chairman of the Global ICT Standardization Forum for India, established in 2009. GISFI has the purpose of increasing the collaboration between European, Indian, Japanese, North-American and other worldwide standardization activities in the area of Information and Communication Technology (ICT) and related application areas. He has been honored by the University of Rome "Tor Vergata", Italy as a Distinguished Professor of the Department of Clinical Sciences and Translational Medicine on March 15, 2016. He is Honorary Professor of University of Cape Town, South Africa, and University of KwaZulu-Natal, South Africa. He has received Ridderkorset af Dannebrogordenen (Knight of the Dannenberg) in 2010 from the Danish Queen for the internationalization of top-class telecommunication research and education. He has received several international awards such as IEEE Communications Society Wireless Communications Technical Committee. Recognition Award in 2003 for making the contribution in the field of "Personal, Wireless and Mobile Systems and Networks," Telenor's Research Award in 2005 for impressive merits, both academic and organizational within the field of wireless and personal communication, 2014 IEEE AESS. Outstanding Organizational Leadership Award for: "Organizational Leadership in developing and globalizing the CTIF (Center for TeleInFrastruktur) Research Network,' and so on. He has been Project Coordinator of several EC projects namely, MAGNET, MAGNET Beyond, eWALL and so on. He has published more than 40 books, 1000 plus journal and conference publications, more than 15 patents, over $100 \mathrm{Ph} . \mathrm{D}$. Graduates and a more substantial number of Masters (over 250). Several of his students are today worldwide telecommunication leaders themselves. Under his leadership, magnitudes of close collaborations are established among premier universities across the globe. The partnerships are regulated by guidelines of the Memorandum of Understanding (MoU) between the collaborating universities. 